\title{
Editorial
}

\section{Epidural anesthesia in infants}

$\mathrm{R}$ EGIONAL anesthesia is routinely used in children to provide analgesia in various situations and settings ranging from the perioperative provision of analgesia to pain control in children with cancerous tumours, major trauma or chronic diseases and children with chronic pain. The literature is replete with reports on the clinical benefits and risks of regional blocks in children, ${ }^{1,2}$ the pharmacological parameters of the analgesic agents used for these blocks ${ }^{3-6}$ and the minor and major complications encountered. ${ }^{7,8}$ Over the years the expertise that anesthesiologists have acquired in the field of pediatric regional anesthesia has enabled them to increase the indications and lower the age of the children in which these techniques are applied, and they are routinely placing epidurals in infants.

Because, contrary to adult patients, children will usually not accept to be awake for the block or for surgery, they are first anesthetized or heavily sedated depending on their age and the circumstances. The sequence anesthesia/sedation-regional block is widely accepted in pediatric anesthesia practice as it provides an immobile child, thus safer conditions for the procedure.

A recent report of paraplegia following intracord injection during an attempt to place a thoracic epidural in an adult who was anesthetized and therefore unable to complain of paresthesias ${ }^{9}$ led to the authors' recommendation that anesthesia never precede a regional technique in any patient, regardless of age. This resulted in an immediate and strong reaction from a large group of pediatric anesthesiologists from around the world. ${ }^{10}$ They argued that the authors did not take into account what has become a standard of care in pediatric anesthesia and that this recommendation would discourage the performance of regional techniques in children depriving them of an important analgesic tool.

This controversy had the advantage of demonstrating that, based on the extensive literature available, children are not small adults but a particular group of patients with specific needs and that in the hands of experienced physicians they can benefit from regional anesthesia techniques while anesthetised without increased risk. It was also an opportunity to emphasise the need to select the appropriate regional technique for the patient, the type of surgery and the postoperative analgesia requirements.

Infants are some of the most challenging patients as far as the provision of analgesia is concerned. An infant is immature physiologically and pharmacologically. He metabolises and eliminates local anesthetics and opioid solutions slowly. On the other hand, his pain pathways and centres and the synaptic connections between them are all in place and functioning. $\mathrm{He}$ feels pain and reacts to it by mounting huge comportmental and hormonal responses, all of which are abolished if analgesics are given. Hence, one is faced with the dilemma of providing adequate analgesia with the potential risk of complications or the unacceptable alternative of withholding effective analgesia and leaving a child to suffer.

A number of systems interact to put the infant at risk. His intercostal muscles play an important role in the mechanics of respiration and his diaphragmatic muscles are less resistant to fatigue. The child will be more prone to respiratory depression than an older child if a motor block is high and involves intercostal muscles. On the other hand, pain can restrict adequate respiration and is a powerful vaso-constrictor. An infant's nervous system is still maturing and the blood-brain barrier is not totally developed. An excessive dose or continuous infusion of morphine can result in accumulation of the drug and lead to respiratory depression and convulsions. The rate of hepatic and renal metabolism of analgesics is decreased in infants and elimination of the drugs is slower. Morphine tends to remain for longer periods in the body and therapeutic as well as toxic blood concentrations are reached after administration of lower doses

Address correspondence to: Joëlle F. Desparmet MD, Department of Anaesthesia, Montreal Children's Hospital, 2300 Tupper Strect, Montréal, Québec H3H 1 P3 Phone: 514-934-4463; Fax: 514-934-4341 
given at a longer dosing interval. ${ }^{11}$ Protein binding of local anesthetics is reduced because $\alpha_{1}$-glycoprotein, the main protein to which local anesthetics bind in the blood, is low until nine months of age. ${ }^{12}$ This leaves a greater amount of the free, potentially toxic form of the local anesthetic to enter cardiac and brain cells. On the other hand, the volume of distribution of the drug is greater and peak plasma concentrations tend to be lower in infants than in older children.

With these facts in mind, how can we use epidural anesthesia to provide an infant with safe, effective analgesia? An epidural catheter can be placed while the baby is asleep and a local anesthetic, an opioid or a combination of both administered via the catheter. In experienced hands, an epidural catheter is relatively easily inserted in children of all ages including newborns. Pediatric-size catheters can be placed by a thoracic $^{13}$ or lumbar approach or through the sacral hiatus in infants. In the latter case, the catheter can be advanced cephalad as far as the lumbar and thoracic epidural space. ${ }^{14}$ There is always a risk of the catheter kinking as it is advanced and the problem lies in locating the exact position of the catheter tip. One can confirm the catheter's position by radiographic imaging but this is time consuming and sometimes not easy to interpret. This is why the technique involving electrical stimulation guidance described by Tsui et al. in this journal is such an important innovation. They previously reported this technique but this is the first time it is described in an infant.

The authors use a low electrical current applied through the catheter much as one would through a needle to locate a peripheral nerve. In the present case report, as they advanced the catheter, they stimulated first the lower limb then the abdominal muscles until they reached the desired level which was detected by intercostal movement at very low electrical input. This placed the catheter tip exactly at the level where the analgesic solution needed to be delivered, thereby reducing the amount of analgesic required and maximising analgesia.

Once correct epidural catheter placement has been confirmed, a bolus dose of local anesthetic with or without the adjunction of an opioid is injected, followed by a continuous infusion. While recipes and amount of drugs administered vary from institution to institution, all agree on certain principles. Children should be continuously monitored while receiving an epidural infusion. A maximum local anesthetic dose of $0.4-0.5 \mathrm{mg} \cdot \mathrm{kg}^{-1} \cdot \mathrm{hr}^{-1}$ should not be exceeded in infants and children. ${ }^{14}$ The total daily dose should be decreased by $50 \%$ in newborns compared with that in older children. The concentration of local anesthetic in the continuous infusion should be reduced as higher concentrations do not improve postoperative analgesia but can lead to a motor block and to overdosage.

In an infant a bolus of $0.3-0.5 \mathrm{ml} \cdot \mathrm{kg}^{-1}$ of a solution of bupivacaine $0.25 \%$ with epinephrine $1: 200000$ with or without $1 \mu \mathrm{g} \cdot \mathrm{kg}^{-1}$ fentanyl is a classic pre-incision dose. In the newborn the concentration of the bolus can be decreased in order to obtain an adequate volume of solution without increasing the total dose of local anesthetic. This can be followed by an infusion of bupivacaine $0.1-0.125 \%$ combined with $1 \mu \mathrm{g} \cdot \mathrm{ml}^{-1}$ fentanyl and given at a rate of $0.15-0.2 \mathrm{ml} \cdot \mathrm{kg}^{-1} \cdot \mathrm{hr}^{-1}$ $\left(0.15-0.25 \mathrm{mg} \cdot \mathrm{kg}^{-1} \cdot \mathrm{hr}^{-1}\right.$ of bupivacaine and 0.15 $0.2 \mu \mathrm{g} \cdot \mathrm{kg}^{-1} \cdot \mathrm{hr}^{-1}$ fentanyl). The infusion is started at the low end of the dosing range, then increased as needed without exceeding the maximum daily dose. If at this point analgesia is not optimal, the infusion should be discontinued and another analgesia regimen adopted. An infant who is restless may be showing signs of local anesthetic toxicity rather than exhibiting pain behaviour ${ }^{15}$ so that increasing the rate of the infusion further would only make matters worse.

Infants receiving epidural analgesia should be carefully monitored but at what age children with epidurals can be monitored on a ward as opposed to an intensive setting is dependent on what is available in individual institutions. An accepted monitoring regimen is continuous monitoring in an intensive setting under 3-6 mo of age and first $24 \mathrm{hr}$ in an intensive setting then closely monitored on the ward for older infants. Respiratory rate alone is not a reliable index of respiratory status. Children can have respiratory depression with a normal rate but shallow breathing. Monitoring of infants should include oxygen saturation and possibly apnea monitoring. Most importantly, these monitors are useless without careful, repeated nursing and medical assessment.

Infants can benefit from regional anesthesia. Better postoperative courses and shorter hospital stays have both been demonstrated in children receiving regional anesthesia. Good knowledge of the scientific data, experience in regional techniques in small children and sound judgement are prerequisites. Techniques such as that described by Tsui et al. should improve the safety of these procedures. 


\section{L'anesthésie péridurale chez les nourrissons}

L'anesthésie régionale est utilisée couramment en pédiatrie afin de fournir une analgésie dans des situations et des milieux divers. Qu'il s'agisse de l'analgésie périopératoire ou du soulagement de la douleur chez les enfants atteints de tumeurs cancéreuses, de traumatismes importants ou de maladies chroniques ou d'enfants souffrant de douleurs chroniques. La documentation présente une foule d'articles sur les bénéfices cliniques et les risques des blocs régionaux chez les enfants, ${ }^{1,2}$ sur les paramètres pharmacologiques des agents analgésiques utilisée pour ces blocs ${ }^{3-6}$ et sur les complications mineures et majeures rencontrées. ${ }^{78} \mathrm{Au}$ fil des ans, l'expérience acquise par les anesthésiologistes dans le domaine de l'anesthésie régionale pédiatrique leur a permis de multiplier les indications et d'appliquer ces techniques chez des enfants de plus en plus jeunes. Maintenant, ils utilisent régulièrement des cathéters périduraux chez les nourrissons.

Comme les enfants, contrairement aux adultes, n'acceptent pas habituellement d'être éveillés pendant le bloc ou l'intervention chirurgicale, ils reçoivent d'abord une anesthésie ou une sédation lourde adaptée à leur âge et aux circonstances. La séquence anesthésie/sédation-bloc régional est largement acceptée dans l'exercice de l'anesthésie pédiatrique, car elle assure l'immobilité de l'enfant et, par conséquent, des conditions sécuritaires pour l'utilisation de la technique anesthésique.

Un cas récent de paraplégie à la suite d'une injection intramédullaire pendant une tentative de mise en place d'une aiguille épidurale thoracique, chez un adulte qui avait été anesthésié et était donc incapable de se plaindre de paresthésies ${ }^{9}$, a donné lieu aux recommandations des auteurs à l'effet que l'anesthésie ne devrait jamais précéder une technique régionale chez aucun patient, quel que soit son âge. Cela a donné lieu à une réaction immédiate et énergique d'un grand nombre d'anesthésiologistes pédiatriques du monde entier. ${ }^{10}$ Ils ont déclaré que les auteurs n'avaient pas tenu compte de ce qui était devenu un standard de soins en anesthésie pédiatrique et que cette recommandation allait décourager la réalisation de techniques régionales chez les enfants, privant ces derniers d'un outil analgésique important.

Cette controverse a eu l'avantage de démontrer que, sur la base de l'imposante documentation offerte, les enfants ne sont pas des petits adultes mais un groupe particulier de patients qui ont leurs besoins spécifiques et qu'ils peuvent bénéficier, ayant été endormis, de techniques régionales d'anesthésie utilisées par des médecins expérimentés, sans risques accrus. C'était aussi l'occasion de mettre l'accent sur la nécessité de sélectionner la technique régionale appropriée au patient, le type d'intervention chirurgicale et les besoins d'analgésie postopératoire.

Les nourrissons sont parmi les patients qui représentent le plus grand défi quant à l'administration de l'analgésie. Le bébé n'a pas encore atteint sa maturité physiologique et pharmacologique. Il métabolise et élimine lentement les anesthésiques locaux et les solutions opiö̈des. Par ailleurs, les voies et les centres de la douleur ainsi que les connexions synaptiques entre eux sont déjà en place et en fonction. Il ressent la douleur et y réagit par de vives réponses hormonales et compartimentales, réactions pourtant abolies si des analgésiques sont administrés. Nous sommes alors confrontés au dilemme de fournir une analgésie adéquate qui comporte un risque potentiel de complications ou, option inacceptable, de refuser l'analgésie efficace et de laisser souffrir l'enfant.

De nombreux systèmes interagissent dans le fait d'exposer le nourrisson. Ses muscles intercostaux jouent un rôle important dans le mécanisme de la respiration et ses muscles diaphragmatiques sont moins résistants à la fatigue. Le bébé est davantage sujet à la dépression respiratoire que l'enfant plus âgé si le blocage moteur est haut situé et implique les muscles intercostaux. Par contre, la douleur peut limiter une respiration adéquate et être un puissant vasoconstricteur. Le système nerveux d'un bébé est en pleine maturation et la barrière hémato-encéphalique n'est pas complètement développée. Une dose excessive ou une perfusion continue de morphine peut provoquer une accumulation de médicament et entraîner une dépression respiratoire et des convulsions. Le taux métabolique hépatique et rénal des analgésiques est plus faible chez les bébés et l'élimination des médicaments est plus lente. La morphine a tendance à demeurer plus longtemps dans l'organisme et des concentrations sanguines thérapeutiques aussi bien que toxiques sont atteintes après l'administration de faibles doses données à des intervalles plus longs entre deux doses successives. ${ }^{11}$ D'une part, la liaison des anesthésiques locaux aux protéines est réduite parce que l' $\alpha$ l-glycoprotéine, protéine principale à laquelle les anesthésiques locaux se lient dans le sang, est peu abondante avant l'âge de neuf mois. ${ }^{12} \mathrm{Ce}$ qui laisse une plus grande quantité d'anesthésique local libre, de forme potentiellement toxique, d'entrer dans les cellules cardiaques et cérébrales. D'autre part, le volume de distri- 
bution du médicament est plus grand et les concentrations plasmatiques maximales tendent à être plus faibles chez les bébés que chez les enfants plus âgés.

En pensant à ces faits, comment peut-on utiliser l'anesthésie péridurale chez le nourrisson et assurer une analgésie sécuritaire et efficace? Un cathéter épidural peut être placé pendant que le bébé est endormi et un anesthésique local, un opioïde ou une combinaison des deux médicaments, administré par le cathéter. Une personne d'expérience peut insérer facilement un cathéter épidural chez des enfants de tous âges, y compris des nouveau-nés. Chez les bébés, les cathéters de dimension pédiatrique peuvent être insérés par voie thoracique ${ }^{14}$ ou lombaire ou par l'échancrure sacrée. Dans ce dernier cas, le cathéter peut être poussé en direction céphalique aussi loin que dans l'espace épidural lombaire et thoracique. ${ }^{14}$ Il y a toujours un risque de coudure du cathéter à mesure qu'il est inséré et la difficulté réside dans la localisation de la position exacte de la pointe du cathéter. On peut confirmer la position du cathéter par l'imagerie radiographique, ce qui exige du temps et, parfois, les résultats sont difficiles à interpréter. C'est pourquoi la technique de guidage par stimulation électrique, décrite par Tsui et coll. dans le présent numéro du journal, représente une telle innovation. Ils ont déjà mentionné cette technique, mais c'est la première fois qu'ils en décrivent l'usage chez un nourrisson.

Les auteurs utilisent un faible courant électrique appliqué au travers du cathéter, de manière semblable à l'utilisation d'une aiguille de neurostimulation, pour localiser un nerf périphérique. Dans le cas présent, à mesure qu'ils avançaient le cathéter, ils ont stimulé d'abord les membres inférieurs, puis les muscles abdominaux jusqu'à ce qu'ils atteignent le niveau voulu qui a été détecté par un mouvement intercostal provoqué par une stimulation de très faible intensité électrique. Cela a permis de placer la pointe du cathéter au niveau exact où la solution analgésique devait être administrée, réduisant ainsi la quantité d'analgésique requise et optimalisant l'analgésie.

Une fois que la mise en place correcte du cathéter a été confirmée, une dose d'anesthésique local en bolus avec ou sans ajout d'opioïde est injectée, suivie d'une perfusion continue. Même si les régimes et les quantités de médicaments varient d'une institution à l'autre, tous s'accordent sur certains principes. Les enfants devraient être sous constante surveillance pendant qu'ils reçoivent une perfusion épidurale. La dose maximale d'anesthésique local ne devrait pas dépasser $0,4-0,5 \mathrm{mg} \cdot \mathrm{kg}^{-1} \cdot \mathrm{h}^{-1}$ chez les bébés et les enfants. ${ }^{14} \mathrm{La}$ dose quotidienne totale devrait être réduite de $50 \%$ chez les nouveau-nés par rapport aux enfants plus âgés. La concentration d'anesthésique local de la perfusion continue devrait être réduite, puisque des concentrations élevées n'améliorent pas l'analgésie postopératoire mais peuvent provoquer un blocage moteur et un surdosage.

Chez un bébé, un bolus de $0,3-0,5 \mathrm{ml} \cdot \mathrm{kg}^{-1} \mathrm{~d}^{\prime}$ une solution de bupivacaïne à $0,25 \%$ combinée à de l'épinéphrine l:200 000 avec ou sans $1 \mu \mathrm{g} \cdot \mathrm{kg}^{-1}$ de fentanyl constitue la dose classique administrée avant l'incision cutanée. Chez le nouveau-né, la concentration du bolus peut être diminuée en vue d'obtenir un volume suffisant de solution sans augmenter la dose totale d'anesthésique local. On peut administrer ensuite une perfusion de bupivacaïne à $0,1-0,125 \%$ combinée à $1 \mu \mathrm{g} \cdot \mathrm{ml}^{-1}$ de fentanyl selon un débit de $0,15-0,2 \mathrm{ml} \cdot \mathrm{kg}^{-1} \cdot \mathrm{h}^{-1}\left(0,15-0,25 \mathrm{mg} \cdot \mathrm{kg}^{-1} \cdot \mathrm{h}^{-1}\right.$ de bupivacaine et $0,15-0,2 \mu \mathrm{g} \cdot \mathrm{kg}^{-1} \cdot \mathrm{h}^{-1}$ de fentanyl). La perfusion débute avec le régime le plus bas dans l'éventail des doses, puis il est augmenté au besoin sans dépasser la dose quotidienne maximale. Si l'analgésie n'est pas optimale à ce moment, la perfusion doit être stoppée et un autre schéma d'analgésie adopté. Un bébé agité peut afficher des signes de toxicité à l'anesthésique local plutôt qu'une réaction à la douleur ${ }^{15}$, de sorte que l'augmentation du débit de la perfusion ne pourrait faire qu'aggraver la situation.

Les bébés qui reçoivent une analgésie épidurale doivent être sous la surveillance étroite. Toutefois, l'âge auquel les enfants qui ont une analgésic épidurale peuvent être placés sous monitorage à l'unité des soins réguliers, en opposition aux soins intensifs, dépend de ce qui est offert dans chacune des institutions. Un régime admis est un monitorage continu dans une unité de soins intensifs pour des enfants de moins de 3-6 mois et pendant les vingt-quatre premières heures dans une unité de soins intensifs suivies d'une période d'étroite surveillance à l'unité des soins réguliers pour les enfants plus âgés. La fréquence respiratoire seule $n$ 'est pas un indice fiable de l'état respiratoire. Les enfants peuvent avoir une dépression respiratoire et une fréquence normale, mais une respiration superficielle. Le monitorage des nourrissons doit inclure la saturation en oxygène et, probablement, le monitorage de l'apnée. Plus important encore, les moniteurs sont inutiles sans évaluation attentive et répétée par le personnel infirmier et médical.

Les bébés peuvent bénéficier de l'anesthésie régionale. Une meilleure évolution postopératoire et un séjour hospitalier plus court ont été observés chez des enfants qui ont reçu une anesthésie régionale. Une connaissance solide des données scientifiques, une expérience technique de l'anesthésie régionale chez de jeunes enfants et un bon jugement sont des prérequis 
à son utilisation. Des techniques comme celle que décrivent Tsui $e t$ coll. peuvent améliorer la sécurité des interventions.

\section{References}

1 Strafford $M A$, Wilder RT, Berde $C B$. The risk of infection from epidural analgesia in children: a review of 1620 cases. Anesth Analg 1995; 80: 234-8.

2 Berde C. Regional anesthesia in children: what have we learned? (Editorial) Anesth Analg 1996; 83: 897-900.

3 Eyres $R L$, Hastings $C L$, Brown TCK, Oppenbeim RC. Plasma bupivacaine concentrations following lumbar epidural anaesthesia in children. Anaesth Intensive Care 1986; 14: 131-4.

4 Desparmet J, Meistelman C, Barre J, Saint-Maurice C. Continuous epidural infusion of bupivacaine for postoperative pain relief in children. Anesthesiology 1987; 67: 108-12.

5 McCloskey JJ, Haun SE, Deshpand JK. Bupivacaine toxicity secondary to continuous caudal epidural infusion in children. Anesth Analg 1992; 75: 287-93.

6 Laarson BA, Lönnqvist PA, Olsson GL. Plasma concentration of bupivacaine in neonates after continuous epidural infusion. Anesth Analg 1997; 84: 501-5.

7 Giaufré E, Dalens B, Gombert A. Epidemiology and morbidity of regional anesthesia in children: a one-year prospective survey of the French-language Society of Pediatric Anesthesiologists. Anesth Analg 1996; 83: 904-12.

8 Goldman LJ. Complications in regional anesthesia. (Editorial) Pediatr Anaesth 1995; 5: 3-9.

9 Bromage PR, Benumof JL. Paraplegia following intracord injection during attempted epidural anesthesia under general anesthesia. Reg Anesth Pain Med 1998; 23: 104-7.

10 Krane EJ, Dalens BJ, Murat I, Murrell D. The safety of epidurals placed during general anesthesia. (Editorial) Reg Anesth Pain Med 1998; 23: 433-8.

11 Koren $G$, Butt $W$, Chinyanga $H$, Soldin $S$, Tan $Y K$, Pape $K$. Postoperative morphine infusion in newborn infants: assessment of disposition characteristics and safety. J Pediatr 1985; 107: 963-7.

12 Wood $M$, Wood $A J J$. Changes in plasma drug binding and alpha 1 -acid glycoprotein in mother and newborn. Clin Pharmacol Ther 1981; 29: 522-6.

13 Tobias JD, Lowe S, O'Dell N, Holcomb GW III. Thoracic epidural anaesthesia in infants and children. Can J Anaesth 1993; 40: 879-82.

14 Bosenberg AT, Bland BAR, Schulte-Steinberg O, Downing $J W$. Thoracic epidural anesthesia via caudal route in infants. Anesthesiology 1988; 69: 265-9.

15 Berde $C B$. Convulsions associated with pediatric regional anesthesia. (Editorial) Anesth Analg 1992; 75: 164-6. 